\title{
SINGULAR HAMMERSTEIN EQUATIONS AND MAXIMAL MONOTONE OPERATORS
}

BY H. BRÉZIS AND F. E. BROWDER

Communicated by Alberto P. Calderón, April 8, 1976

Consider the nonlinear integral equation of Hammerstein type

$$
u(x)+\int_{\Omega} k(x, y) f(y, u(y)) \beta(d y)=h(x) \quad(x \in \Omega),
$$

where $h$ and the solution $u$ lie in a space $X$ of measurable functions on $\Omega$. The Hammerstein equation is said to be regular if for

$$
\text { (2) } F(u)(y)=f(y, u(y)) \quad(y \in \Omega) ; \quad K v(x)=\int_{\Omega} k(x, y) v(y) \beta(d y) \quad(x \in \Omega) \text {, }
$$

the operator $K F$ is defined on all of $X$, and singular otherwise.

In some recent papers (summarized in [2]), the writers have studied the existence theory for regular Hammerstein equations in $L^{p}(\beta)$ with $1<p \leqslant+\infty$ under very general assumptions on $K$ and $F$. In later papers (cf. [4]), one of the writers has obtained general existence results for the singular case, using measuretheoretic arguments and mild compactness assumptions on $K$. We present results here without compactness assumptions based on a new theorem on linear monotone operators.

THEOREM 1. Let $X$ be a reflexive Banach space, $L_{0}$ and $L_{1}$ linear monotone mappings from $X$ into $2^{X^{*}}$ with $L_{0} \subseteq L_{1}^{*}$. Then there exists a maximal monotone linear map from $X$ into $2^{X^{*}}$ such that $L_{0} \subseteq L \subseteq L_{1}^{*}$.

For single-valued, densely defined maps in Hilbert space, this coincides with a theorem of R. S. Phillips [6] obtained using ideas of M. Kreìn [5]. For reflexive Banach spaces, in general, we have as a corollary a result obtained in 1968 by one of the writers [1]:

THEOREM 2. Let $X$ be a reflexive Banach space, $L$ a closed linear monotone map from $X$ into $2^{X^{*}}$. Then $L$ is maximal monotone if and only if $L^{*}$ is monotone.

We sketch the proof of Theorem 1 (detailed proofs are given in [3]). By a Zorn's Lemma argument we may construct a monotone linear map $L$ with $L_{0}$ $\subseteq L \subseteq L_{1}^{*}$ such that $L$ is maximal monotone in the graph of $L_{1}^{*}$. Let $J$ be a duality map of $X$ into $X^{*}$ corresponding to a norm on $X$ with $X$ and $X^{*}$ locally uniformly convex.

AMS (MOS) subject classifications (1970). Primary 47H05, 47G05; Secondary 47H15. 
Let $w_{0}$ be any element of $X^{*}$. It suffices to find $u_{0}$ in $X$ such that $w_{0}$ $\in(L+J)\left(u_{0}\right)$. For each finite-dimensional subspace $M$ of $X$, let $\xi_{M}$ be the injection map of $M$ into $X, \xi_{M}^{*}: X^{*} \rightarrow M^{*}$. We form linear monotone mappings $L_{M}$ and $L_{1, M}$ of $M$ into $2^{M^{*}}$ with $L_{M} \subseteq\left(L_{1, M}\right)^{*}$ by

$$
L_{M}(x)=\xi_{M}^{*}(L(x)), \quad L_{1, M}(x)=\xi_{M}^{*}\left(L_{1}(x)\right) .
$$

We apply the multivalued finite-dimensional version of Phillips' theorem (a simple direct proof for which is given in [3]) to obtain a maximal monotone mapping $K_{M}$ from $M$ to $2^{M^{*}}$ such that $L_{M} \subseteq K_{M} \subseteq\left(L_{1, M}\right)^{*}$. Hence, we may find $u_{M}$ in $M$ such that $\xi_{M}^{*}\left(w_{0}\right) \in K_{M}\left(u_{M}\right)+\xi_{M}^{*}\left(J\left(u_{M}\right)\right)$.

For each $[u, w]$ in $G\left(L_{1}\right)$ and for each $[x, y]$ in $G(L)$ with $u$ and $x$ in $M$,

$$
\begin{gathered}
\left\langle w_{0}-J\left(u_{M}\right), u\right\rangle=\left\langle w, u_{M}\right\rangle, \\
\left\langle y+J\left(u_{M}\right)-w_{0}, x-u_{M}\right\rangle \geqslant 0 .
\end{gathered}
$$

The elements $\left\{\left[u_{M}, J\left(u_{M}\right)\right]\right\}$ are bounded since $J$ is coercive. Since $X$ is reflexive, we may assume a filter $\left\{\left[u_{M}, J\left(u_{M}\right)\right]\right\}$ converging weakly to $\left[u_{0}, y_{0}\right]$ in $X$ $\times X^{*}$. Since equality (3) holds eventually for each $[u, w]$ in $G\left(L_{1}\right)$, we may take the limit to find that $\left\langle w_{0}-y_{0}, u\right\rangle=\left\langle w, u_{0}\right\rangle$ for all $[u, w]$ in $G\left(L_{1}\right)$. Hence $\left[u_{0}, w_{0}-y_{0}\right]$ lies in $G\left(L_{1}^{*}\right)$. From inequality (4) which holds eventually for each $[x, y]$ in $G(L)$, we obtain

$$
\varlimsup\left\langle J\left(\left(u_{M}\right), u_{M}\right)\right\rangle-\left\langle y_{0}, u_{0}\right\rangle \leqslant\left\langle y+y_{0}-w_{0}, x-u_{0}\right\rangle .
$$

Since $J$ is pseudo-monotone, the left side is nonnegative. Since $\left[u_{0}, w_{0}-y_{0}\right]$ $\in G\left(L_{1}^{*}\right)$ and $L$ is assumed maximal monotone in $G\left(L_{1}^{*}\right),\left[u_{0}, w_{0}-y_{0}\right]$ lies in $G(L)$. Replacing $[x, y]$ by this element, it follows that the left side of $(5)$ is zero, and hence $y_{0}=J\left(u_{0}\right)$. Thus $w_{0}-J\left(u_{0}\right) \in L\left(u_{0}\right)$, i.e. $w_{0} \in(L+J)$ $\left(u_{0}\right)$. Q.E.D.

The application to singular Hammerstein equations is made through the following more general theorem:

THEOREM 3. Let $\beta$ be a finite measure on $\Omega, X$ a reflexive Banach space with $L^{\infty}(\beta) \subseteq X \subseteq L^{1}(\beta), L^{\infty}(\beta) \subseteq X^{*} \subseteq L^{1}(\beta)$. Let $F$ be a hemicontinuous, monotone angle-bounded map of $X$ into $X^{*}$ with $0 \in \operatorname{Int}(R(F))$. Let $K$ be a bounded linear map of $L^{1}(\beta)$ into $L^{1}(\beta)$ with $\langle K v, v\rangle \geqslant 0$ for all $v$ in $L^{\infty}(\beta)$ Then for each $h$ in $X$, there exists $u$ in $X$ such that $u+K F(u)=h$ and $\langle K v-$ $K F(u), v-F(u)\rangle \geqslant 0$ for all $v \in L^{\infty}(\beta)$ with $K v \in X$.

To prove Theorem 3 , we may set $h=0$ by a change of variables. Let $L_{1}$ be the mapping from $X^{*}$ to $X$ with effective domain $L^{\infty}(\beta)$ and with $L_{1}(v)=$ $K^{\prime}(v)$ where $K^{\prime}: L^{\infty}(\beta) \rightarrow L^{\infty}(\beta)$ is the dual of $K$. Then $L_{1}$ is monotone and $L_{1}^{*}$ is a restriction of $K$. Let $K^{\#}$ be the mapping from $X^{*}$ to $X$ with domain $D\left(K^{\#}\right)=\left\{v \in L^{\infty}(\beta)\right.$ and $\left.K v \in X\right\}$ and $K^{\#} v=K v$. Since $K^{\#} \subset L_{1}^{*}$ we may find 
by Theorem 1, a maximal monotone operator $L$ satisfying $K^{\#} \subseteq L \subseteq L_{1}^{*}$. Finally one solves $0 \in L^{-1}(u)+F(u)$.

\section{BIBLIOGRAPHY}

1. H. R. Brézis, On some degenerate parabolic equations, Proc. Sympos. Pure Math., vol. 18, part 1, Amer. Math. Soc., Providence, R. I., 1970, pp. 28-38. MR 42 \#8346.

2. H. Brézis and F. E. Browder, Nonlinear integral equations and systems of Hammerstein type, Advances in Math. 18 (1975), 115-147.

3. - Linear maximal monotone operators and singular nonlinear integral equations of Hammerstein type, E. Rothe Festschrift (to appear).

4. F. E. Browder, Strongly nonlinear integral equations of Hammerstein type, Proc. Nat. Acad. Sci. U.S.A. 72 (1975), 1937-1939.

5. M. G. Kreìn, The theory of self-adjoint extensions of semi-bounded hermitian transformations and its applications. I, II, Mat. Sb. N.S. 20 (62) (1947), 431-495; ibid, 21 (63) (1947), 365-404. (Russian) MR 9, 515.

6. R. S. Phillips, Dissipative operators and hyperbolic systems of partial differential equations, Trans. Amer. Math. Soc. 90 (1959), 193-254. MR 21 \#3669.

DEPARTMENT OF MATHEMATICS, UNIVERSITY OF CHICAGO, CHICAGO, ILLINOIS 60637 